\title{
The visual status of adolescents in Riyadh, Saudi Arabia: a population study
}

This article was published in the following Dove Press journal:

Clinical Ophthalmology

\author{
Ali Alsaqr \\ Ali Abu Sharha \\ Raied Fagehi \\ Awatif Almutairi \\ Sarah Alosaimi \\ Abdulrahman Almalki \\ Abdulaziz Alluwaymi \\ Department of Optometry, \\ College of Applied Medical Sciences, \\ King Saud University, Riyadh, \\ Saudi Arabia
}

Purpose: The visual status of adolescents in Saudi Arabia (SA) has not been well reported. To date, the prevalence and types of refractive errors (REs), amblyopia, strabismus, and correctable visual impairments have not been quantified. The aim of the study was to investigate the visual status in adolescents in Riyadh, SA.

Methods: This study was based on a population cross-sectional and random cluster design. After design and the sample calculations, 1,007 participants, 12-20 years of age, were screened during the study. Nine participants were excluded due to ocular disorders. The participants were assessed for REs, distance visual acuity logarithm of the minimum angle of resolution, contrast sensitivity, stereoacuity, pinhole test findings, and cover-uncover test findings.

Results: The results showed that $55.5 \%$ of the participants had some form of REs, while correctable visual impairment was found in one-fifth of the screened participants. Myopia was the dominant type (53.3\%, ranged from -0.50 DS to -14.00 DS), whereas hyperopia was found in $2.2 \%(+2.00 \mathrm{DS}$ to $+5.50 \mathrm{DS})$ and astigmatism was present in $15 \%(-0.75 \mathrm{DC}$ to $-5.25 \mathrm{DC})$. Only $43 \%$ of the participants had corrected REs; however, the noncompliance for spectacle use was $20.25 \%$.

Conclusion: This study was the first attempt to investigate the visual status in adolescents in SA. It provided estimations of the REs, amblyopia, and strabismus. The high prevalence of REs emphasizes the need to identify the best proactive strategies to detect and manage REs to reduce the incidence of visual impairment in SA. Increasing awareness about eye health and employing efficient screening programs could help to address the need for REs corrections.

Keywords: amblyopia, myopia, refractive errors, Riyadh, Saudi, strabismus, visual impairment

\section{Introduction}

Approximately 2.3 billion people globally have impaired vision due to refractive error (RE). ${ }^{1}$ The number of people with visual impairments due to uncorrected RE (URE) was predicted to be 101.2 million in 2010 , which was a $15 \%$ increase since $1990 .^{2}$ This number is suggested to double by 2020 as a result of avoidable causes, such as URE, cataracts, and vitamin A deficiency, suggesting the urgent need for more efforts to avoid this crisis. ${ }^{3}$ URE has economic and social implications on the quality of life, with global economic damages estimated at $\sim \$ 269$ billion per annum because of lost productivity. ${ }^{4,5}$

The RE has been linked to physiological and environmental factors, such as near work distances and time spent performing outdoor activities. ${ }^{6-8}$ The prevalence of myopia has increased worldwide, reaching levels as high as $80 \%$ of the population in some Asian countries. ${ }^{9-12}$ In 2010, a global myopia prevalence of 2 billion people was reported. If these trends continue, $50 \%$ of the world population in 2050 will be myopic. ${ }^{13}$
Correspondence: Ali Alsaqr

Department of Optometry, College

of Applied Medical Sciences, King Saud University, PO Box 10219, Riyadh II433, Saudi Arabia

Tel +966114693537

Fax +96 6II 4693536

Email aalsaqr@ksu.edu.sa (c) (1) (5) 2018 Alsaqr et al. This work is published and licensed by Dove Medical Press Limited. The full terms of this license are available at https://www.dovepress.com/terms.php
and incorporate the Creative Commons Attribution - Non Commercial (unported, v3.0) License (http://creativecommons.org/licenses/by-nd/3.0/). By accessing the work you BY NC and incorporate the Creative Commons Attribution - Non Commercial (unported, v3.0) License (http:///creativecommons.org/licenses/by-nc/3.0/). By accessing the work you
hereby accept the Terms. Non-commercial uses of the work are permitted without any further permission from Dove Medical Press Limited, provided the work is properly attributed. For permission for commercial use of this work, please see paragraphs 4.2 and 5 of our Terms (https://www.dovepress.com/terms.php). 
The worldwide RE prevalence has marked differences across geographical locations, due to racial, age, gender, and ethnic factors. These differences have an influence on the strategies used to manage URE. ${ }^{5}$ Furthermore, RE treatment is a priority in the global initiative for the elimination of avoidable blindness (VISION 2020: The Right to Sight). However, no effective strategy can be implemented without knowing the exact extent of the disorder. Only a few studies have assessed the visual status in Saudi children in the past decades. ${ }^{14-17}$ Moreover, they have included participants only from preschools and primary schools. ${ }^{14-17}$ Population-based studies in adolescents, 12-20 years of age, are important for proactive planning, implementation of suggestions, and monitoring protocols and/or interventions to eliminate avoidable visual impairment in this very productive age group. To the best of our knowledge, this is the first study that aims to investigate the visual status of adolescents in Riyadh, Saudi Arabia (SA).

\section{Methods}

\section{Compliance with ethical standards}

All procedures performed in this study were in accordance with the ethical standards of the institutional and/or national research committee and with the 1964 Declaration of Helsinki and its later amendments or comparable ethical standards. Institutional ethical approval was obtained from the Ethics for Scientific Research Committee, King Saud University. Approval was also obtained from the Ministry of Education and the school headmasters. A written informed consent was obtained from all individual participants' parents included in the study, as well as verbal consent from the participants.

\section{Study design and participants}

The present study followed a cross-sectional population design. The sample population consisted of students attending elementary and high schools, who were $12-20$ years of age.

A structured survey concerning the general and ophthalmic history of the participants and their families was collected. The survey included information about the family's general and ocular history as well as their social characteristics, which included whether the parents are relatives or not, the parents' education, the participant's order in the family, and does any of their sibling have ocular abnormality. Moreover, structured detailed information was collected about the participants' general and ocular history, their lifestyle habits (environmental factors), and the date of the last visit to an eye specialist. However, the environmental factors collected and their impact on the visual status is not presented in this manuscript; however, it is intended to be reported in the future. Most of these questions were in dichotomous format, where more information is required if the answer was yes.

\section{Sample calculation and identification}

The sample size was calculated using the Epi-Info, version 7 (Centers for Disease Control and Prevention, Atlanta, GA, USA; http://www.cdc.gov/epiinfo/7/). A multistage cluster sampling method was used, where 20 highly populated schools from different parts of Al-Riyadh city were randomly selected and the classes included were also randomly selected. In details, these criteria were the inputs in the EpiInfo program. The number of students in both intermediate and high schools was $\sim 470,000$ (obtained from the Riyadh General Directorate of Education, March 2016). The prevalence of RE was estimated at $10 \%-25 \%$ based on previous studies in this geographical region. ${ }^{18}$ The margin of error was set at 5\% (95\% confidence), the design effect was set at 2 , and the clusters equal to 20 . The overall minimal sample required was 644 participants. In each school, a designated area was provided by the school where all the tests for all the students where conducted; the team visiting the school made sure that the venue was safe and suitable for the students and that it was a well-lit room.

The assessed measures were as follows: visual acuity (VA), RE, ocular deviation, stereoacuity, contrast sensitivity (CS), strabismus, amblyopia, and correctable visual impairment.

\section{VA measurement}

The VA measurements were recorded monocularly at a distance of $4 \mathrm{~m}$ using the logarithm of the minimum angle of resolution (logMAR) VA chart (the non-illuminated Early Treatment Diabetic Retinopathy Study chart with Sloan letters [Precision Vision, La Salle, IL, USA]). First, the VA was measured without correction, then with the participants' habitual corrections, if applicable. VA of the right eye was always measured first, followed by VA of the left eye. Testing began with the smallest line that could be seen, and the participant was instructed to read as many letters as possible in the next row. The VA was recorded in terms of the smallest line identified, the scores excluded the letters not identified in the line, or included the number of letters identified in addition to the line.

\section{RE measurement}

The RE was measured without a cycloplegic agent using a handheld autorefractor (ARK-30; Nidek, Gamagori, Japan). The ARK-30 automatically started measuring the RE when it 
was in the right position according to centering and focusing indicators. The same autorefractor was used for all participants throughout the study. As a proactive measure, if the autorefractor did not provide reliable readings for any reason, a distance retinoscopy was conducted. In addition, retinoscopy was performed in a dark room with the participant sitting comfortably at a convenient working distance for both the participants and the examiners. The working distance power was added as a minus power in the trial frame. The participant was instructed to maintain the fixation at a non-accommodative distant target. However, the retinoscopy procedure was not needed and ARK-30 was performed to all participants.

\section{Pinhole acuity}

The pinhole acuity test was used in participants who were unable to reach the $0.00 \log$ MAR line $(6 / 6)$. We examined the pinhole acuity to indicate for any improvement in acuity and the presence of amblyopia. Once the pinhole was conducted, the outcome of the autorefractor was placed in a trial frame and the VA was monocularly measured in the same procedure stated earlier to document any improvement in VA (correctable visual impairment). For those students who had RE, a letter was sent to the parents to inform them of their child's condition and suggesting a visit to eye care professional for a comprehensive eye exam.

\section{Stereoacuity measurement}

The stereoacuity was used to determine the participant's depth perception and ability to fuse stereoscopic targets. We measured stereoacuity using the Randot stereo test, which is a subjective test designed for a quick assessment of the presence and degree of stereopsis. The test consisted of contoured circles at 10 levels of disparity (ranging from 20 to 400 seconds of arc at $40 \mathrm{~cm}$ ) and provided a finely graded sequence. Within each of the 10 targets were 3 circles. Only one of the circles had crosses disparity and would stand out. The examiner held the test perpendicular to the line of sight at $40 \mathrm{~cm}$ in a well-illuminated room with the patient wearing crossed polarized glasses over the correction if habitually used. The participants were asked not to tilt their head. They were then asked which one seemed to float forward or appeared different from the other 2 circles. If one is missed, the examiner tested the preceding line to determine whether the participant could see it or was guessing. The level of stereopsis of the last one accurately identified was noted. The main aim of using the Randot test was to help in indicating the presence of amblyopia. Impaired stereopsis under binocular conditions has been reported to be the most common deficit associated with amblyopia. ${ }^{19}$

\section{CS measurement}

The monocular CS was measured using the MARS CS test (numerical version; Mars Perceptrix Corporation, Chappaqua, NY, USA). The chart was held at $50 \mathrm{~cm}$ and the illumination was set at $\sim 85 \mathrm{~cd} / \mathrm{m}^{2}$. The participants were instructed to read from top to bottom, scoring $0.04 \log C S$ units for each correct letter. The test was stopped if the participant made 2 errors in a row.

\section{Ocular deviation assessment}

Ocular deviation was assessed using the cover-uncover test, with the correction, if any, both at $3 \mathrm{~m}$ and at $40 \mathrm{~cm}$. The participant was asked to look straight ahead at a letter at $3 \mathrm{~m}$, well above their VA threshold. For near testing, the participant was asked to fixate at a pencil pen. The test started with occluding the left eye for 3 seconds. The observer looked for any correcting movement of the uncovered eye. After testing the right eye, the left eye was tested in a similar manner. If there was no manifest misalignment of either eye, the cover was moved back and forth between both eyes, with 1-2 seconds between movements.

\section{Definitions of variables}

The RE was expressed as the spherical equivalent RE (SERE). The RE cut points suggested in the RE survey in children (RESC) protocol was followed. This protocol has been widely used and was also suggested by the World Health Organization. ${ }^{20-26}$ The criteria for each variable are described in Table 1.

\section{Data analysis}

The data analysis included $99.1 \%$ of the collected data. The data were investigated for a normal distribution using the Kolmogorov-Smirnov test, which showed that the data were not normally distributed $(p<0.05)$. Nonparametric tests were, therefore, used where appropriate. The median and interquartile ranges (IQR) were used to report the participants' visual functions. The Spearman's rank correlation test was used to test for data similarity between right and left eye in all variables.

The SERE, unaided VA, and CS were comparable between the right and left eyes. Spearman's rank correlation coefficient revealed a strong relationship between the scores of the right and the left eye (SERE: $r=0.80, p<0.0001$; unaided VA: $r=0.82, p<0.0001$; CS: $r=0.71, p<0.0001$ ). The scores of the visual functions that were reported corresponded to the lowest scores of the 2 eyes. 
Table I Definitions of the criteria used for outcomes cutoff

\begin{tabular}{ll}
\hline Variables & Definitions \\
\hline Myopia & Spherical equivalent refractive error $\leq-0.50 \mathrm{D}$ in one or both eyes \\
Hyperopia & Spherical equivalent refractive error $\geq+2.00 \mathrm{D}$ in one or both eyes \\
Astigmatism & Cylinder power $\geq 0.75 \mathrm{DC}$ if one or both eyes were astigmatic \\
Emmetropia & When neither eye was myopic or hyperopic. \\
Unilateral amblyopia & Interocular difference in the best-corrected VA of 2 lines or more on the chart. \\
Bilateral amblyopia & When the best-corrected binocular VA was abnormal, even after full refractive correction. \\
Correctable visual impairment & Defined as a reduction of the VA of $\geq 0.30$ logMAR (6/I2), when VA improvement of 2 or \\
\hline
\end{tabular}

Abbreviation: VA, visual acuity.

\section{Results}

Of the total 1,007 participants, $9(0.9 \%)$ were excluded for undergoing an eye surgery or because of a previous ocular pathology. In the remaining 998 participants, the age ranged from 12-20 years (median IQR $=16$ [3] years). There were 651 females and 337 male participants in the final study.

\section{Background characteristics}

In total, 525 participants passed their school exams with excellent grades (53.1\%), 446 participants $(45.1 \%)$ with very good or good grades, and the remaining $18(1.8 \%)$ with passing grades. Furthermore, $17.9 \%$ were the first child, $17.9 \%$ were the second child, $18.5 \%$ were the third child, and $45.6 \%$ were greater than the third child. Moreover, $67 \%$ of the participants had RE in their family. The fathers of $\sim 26 \%$ of the participants had RE; the mothers of $9 \%$, and the siblings of $25 \%$ of the participants had RE. Interestingly, first-degree relatives of $42 \%$ of the participants had myopia. In terms of the parents' education, the most frequent highest degree obtained by the mothers was high school (48.5\%), and that obtained by the fathers was a bachelor's degree (36.5\%). However, 53 mothers (5.2\%) and 20 fathers (2\%) had no school certification.

\section{Visual functions of the participants}

The participants' unaided VA was a median of 0.0 and IQR of 0.20 (range, $-0.01 \log$ MAR [6/5] to $2.00 \log$ MAR [6/120]), while the aided VA was $0.00[-0.14][6 / 6,6 / 5]$ and ranged from $0.00(6 / 6) \log$ MAR to $1.00 \log$ MAR (6/60). Based on the SERE, out of the 998 participants, 55.5\% had RE (555 out of 998). The most frequently diagnosed RE was myopia, with 53.3\% (533 participants) being myopic (ranged from -0.50 DS to $-14.00 \mathrm{DS}$ ) and $2.2 \%$ (22 participants) being hyperopic (+2.00 DS to +4.75 DS). It has also been found that $15 \%$ (150 participants) were astigmatic ( $-0.75 \mathrm{DC}$ to $-5.25 \mathrm{DC}$ ) (Table 2). Of all the $555 \mathrm{RE}$ cases, less than half of them (43.6\%) had correcting spectacles (242 participants).
The noncompliance for spectacle use was $20.25 \%$ (49 out of the 242 participants). Moreover, the correctable visual impairment (VA $\geq 0.30 \log M A R$ 6/12) was present in $17.8 \%$ of the participants.

Strabismus was found in 19 (1.9\%) of the participants, and the estimated amblyopia was found in 59 (5.8\%) of the participants. Unilateral amblyopia was estimated at $3.9 \%$ and was more frequent than the estimated bilateral amblyopia (1.9\%). Stereopsis was also tested and the depths perceived ranged from 0 to 200 seconds of arc (median: 32; IQR: 30). The CS of each eye was also among the tests that were performed with a median of $1.6 \mathrm{log}$; IQR: 0.04 (range, $0.12-1.84 \log$ ).

Finally, out of the 998 participants, 450 (45.1\%) had never visited an optometrist; 295 (29.56\%) had not tested their vision for $>1$ year before the study. The parents of the majority of the participants who had never undergone a visual examination either had no formal education or had high school certificates (63.55\%).

\section{Discussion}

The prevalence of RE in school-age children was investigated in several parts of the world. ${ }^{9111,18,28-33}$ The majority of these studies have used the RESC protocol, which involved cycloplegia. It has been suggested previously that auto-refractors without cycloplegia have at least $0.50 \mathrm{D}$ myopic shift. Not using cycloplegia could be a limitation of the current study. However, we were not able to incorporate the cycloplegia in our protocol due to the limited resources available and due to

Table 2 Types, frequencies, and severities of the RE of the participants

\begin{tabular}{|c|c|c|c|}
\hline RE & $\begin{array}{l}\text { Mild } \\
(\text { RE } \leq 3 \mathrm{D})\end{array}$ & $\begin{array}{l}\text { Moderate } \\
(3 \mathrm{D}<\mathrm{RE} \leq 6 \mathrm{D})\end{array}$ & $\begin{array}{l}\text { High } \\
\text { (RE >6 D) }\end{array}$ \\
\hline Myopia & 422 (47.90\%) & $98(9.8 \%)$ & $13(1.3 \%)$ \\
\hline Hyperopia & 14 (1.40\%) & $7(0.7 \%)$ & 0 \\
\hline Astigmatism & 143 (14.3\%) & $7(0.70 \%)$ & 0 \\
\hline
\end{tabular}

Abbreviations: D, diopters; RE, refractive error. 
our expectation that our request would be faced with consent refusal from the vast majority of the parents. This might be because the parents believe that such a procedure would harm their child's eyes. Attempts have been made previously to conduct a cycloplegic RE screening; however, the vast majority of the parents did not approve such a procedure (personal communication). However, the parents might consent for the use of cycloplegia on their children but under the condition of conducting the screening in a hospital which they think is more of a controlled environment. We did not have transportation facility to take the students to a hospital and did not have access to a hospital facility. In this study, we have tried to extend the examination of visual status in residents of SA to cover a wide range of school students; therefore, we needed to conduct this study to provide estimations of the RE prevalence as its magnitude was not previously known. Moreover, although this study did not typically conform with the RESC protocol (specifically, cycloplegia and comprehensive eye exam), it followed their classification of the RE to facilitate comparison with previous and future studies.

URE is the leading cause of visual impairment and the second leading cause of blindness worldwide is associated with unemployment and decreased quality of life. ${ }^{2,34}$ In $\mathrm{SA}, \mathrm{RE}$ is the leading cause of visual impairment $(36 \%),{ }^{35}$ a condition that can be easily treated and managed. In this study, correctable visual impairment was highly prevalent, and was observed in $17.8 \%$ of the participants.

About half of the participants had RE, with most of these participants having myopia. About two-thirds of the participants' relatives had RE, $42 \%$ were myopic, which may have accounted for the high prevalence of RE and myopia. Moreover, half of the participants with RE were corrected with spectacles, almost $20 \%$ of them did not wear their spectacles.

The prevalence of myopia increases with age,,$^{10,11,36,37}$ which also was found in SA where preschool myopia was found in only $2.5 \%$ of the participants. ${ }^{17}$ Our current study revealed a myopia prevalence of $53.3 \%$ in adolescents. This suggests that myopia increased from preschool to adolescence, which was consistent with the findings of previous reports. ${ }^{10,11,36,37}$

In this study, the myopia was the leading type of RE (53.3\%), and exceeded the prevalence reported in the Caucasian population in the USA (33\%-40\%), Australia (17.7\%), Norway (30\%), and Swedish schoolchildren (49.5\%). ${ }^{9,28-31}$ Furthermore, our results were lower than those observed in Chinese adolescents (54\%), Taiwanese schoolchildren (87\%), and Australians of East Asian ethnicity (59\%). ${ }^{11,29,32}$ Our results were also similar to those found in Sudan, but higher than those reported in Jordan (17\%), Turkey (3.2\%),
Ethiopia (7.7\%-10.2\%), Iran (14.90\%-29.3\%), Tunisia (9.1\%), Morocco (6.1\%), and Egypt (12\%). ${ }^{18,33,38-45}$ The variations in myopia prevalence between the current study and previous reports could be the result of several factors, such as genetics, lifestyle (indoor and outdoor activities), and environmental factors. ${ }^{46-50}$

In the present study, the percentage of parents who had RE was high (40\%). It has been reported that genetic and other environmental factors were associated with the myopia development and progression. ${ }^{50-53}$ Moreover, genetic factors combined with specific environmental factors influenced school myopia. ${ }^{8,54}$ A 24-year longitudinal study was conducted on juvenile-onset myopia of 43 pedigrees to assess the relationship of infantile RE and parental history with juvenile-onset myopia. ${ }^{48}$ They reported that children with 2 myopic parents had a very high risk of myopia (6.42 times) compared with the children with 1 or no myopic parents. ${ }^{48}$

The noncompliance that has been found in this study $(20 \%)$ was also similar to those found in previous reports from different parts of the world, including SA. ${ }^{32,55,56}$ These studies showed that $20 \%-40 \%$ of the participants often did not wear spectacles or were not wearing spectacles with the optimum RE correction. Furthermore, it has been suggested that the constant use of spectacles could improve educational outcomes, ${ }^{57}$ which leads to a significant increase in visionrelated quality of life. ${ }^{58}$ Not using corrective spectacles may result from cultural disincentives and esthetic concerns. ${ }^{59,60}$ Therefore, exploring different approaches to increase spectacle use, such as providing free spectacles, educating students/ parents about the importance of wearing spectacles, and prescribing contact lenses, after assessing the participants' ocular health and hygiene, is strongly suggested.

In this study, the estimated prevalence of amblyopia was $5.8 \%$, which is consistent with the findings of some previous studies conducted in Saudi and other parts of the world ${ }^{15,61}$ but higher than other studies at both the Middle East and the world. 14,17,39,42,62-64 These discrepant results may have resulted from differences in the study population (age group) and the definition used for amblyopia. Furthermore, the targeted age group in the previous studies from SA varied significantly, and none of these studies specifically addressed adolescents. In addition, it has been suggested that prevalence of amblyopia is significantly higher in older age groups. ${ }^{62,65}$ Finally, it should be acknowledged that the comprehensive eye exam was not performed in this study, and therefore, may have overestimated the prevalence of amblyopia. On the other hand, we obtained an estimated baseline for the adolescent group in SA that was not available previously. The prevalence of strabismus (1.9\%) was 
similar to a previous report at the local level (although in different age ranges) ${ }^{15}$ but was also higher ${ }^{14,17,39,42}$ and lower ${ }^{18,38,62-64}$ than other studies at both local and international levels. Approximately one-fifth of our participants had correctable visual impairment (due to URE), which was consistent with previous findings. ${ }^{16,27,66,67}$ However, our findings were higher than those reported in European Caucasian children in Australia. ${ }^{68}$ This discrepancy may be due to the wider age groups of children in the higher prevalence studies, in addition to differing prevalences of myopia and astigmatism with differences in ethnicities and the efficiencies of different health programs.

Approximately $45 \%$ of the participants in the present study never had an optometric evaluation, while the majority of their parents had a high school certificate or were uneducated, raising concerns about their visual health, and suggesting that school vision programs should be established to support healthy children.

\section{Conclusion}

This study showed a high RE prevalence as well as high URE. Prevalence estimate of the different types of RE has provided for the first time in Riyadh, SA. This showed that myopia was the most prevalent REs in adolescents. It showed a reasonably high prevalence of the correctable visual impairment. These findings could raise concerns about the health service coverage, the participants' education performance, and quality of life. Moreover, future studies are required to assess the effect of outdoor/sports activities in SA, because the climate is very dry and harsh. In addition, it will be of interest to know the effects of high-intensity sunlight and a lack of humidity on RE and myopia, especially for people living in a desert environment.

\section{Acknowledgments}

The authors extend their appreciation to the College of Applied Medical Sciences Research Centre and the Deanship of Scientific Research at King Saud University for funding this research.

\section{Disclosure}

The authors report no conflicts of interest in this work.

\section{References}

1. Thulasiraj RD, Aravind S, Pradhan K. Spectacles for the millions addressing a priority of "VISION 2020 - The Right to Sight". Community Ophthalmol. 2003;3:19-21. Available from: http://v2020eresource.org/ content/files/spectacles_oct-03.pdf. accessed on October 2017.

2. Naidoo KS, Leasher J, Bourne RR, et al; Vision Loss Expert Group of the Global Burden of Disease Study. Global vision impairment and blindness due to uncorrected refractive error, 1990-2010. Optom Vis Sci. 2016;93:227-234.
3. Pararajasegaram R. VISION 2020-the right to sight: from strategies to action. Am J Ophthalmol. 1999;128:359-360.

4. Taylor H. Refractive errors: magnitude of the need. Community Eye Health. 2000;13:1-2.

5. Resnikoff S, Pascolinia D, Mariott SP, Pokharel GP. Global magnitude of visual impairment caused by uncorrected refractive errors in 2004 . Bull World Health Organ. 2008;86:63-70.

6. Khandekar R, Al Harby S, Mohammed AJ. Determinants of myopia among Omani school children: a case-control study. Ophthalmic Epidemiol. 2005;12:207-213.

7. Liang C, Yen E, Su J, et al. Impact of family history of high myopia on level and onset of myopia. Invest Ophthalmol Vis Sci. 2004;45: 3446-3452.

8. Landmann A, Bechrakis E. Nature or nurture. Effects of parental ametropia on children's refractive errors. Ophthalmologe. 2013;110: 1179-1184.

9. Vitale S, Sperduto RD, Ferris FL. Increased prevalence of myopia in the United States between 1971-1972 and 1999-2004. Arch Ophthalmol. 2009;127:1632-1639.

10. Williams KM, Bertelsen G, Cumberland P, et al; European Eye Epidemiology (E(3)) Consortium. Increasing prevalence of myopia in Europe and the impact of education. Ophthalmology. 2015;122:1489-1497.

11. Lin LLK, Shih YF, Hsiao CK, Chen CJ. Prevalence of myopia in Taiwanese schoolchildren: 1983 to 2000. Ann Acad Med Singapore. 2004;33:27-33.

12. Wu LJ, You QS, Duan JL, et al. Prevalence and associated factors of myopia in high-school students in Beijing. PLoS One. 2015; 10(3):e1020764.

13. Holden BA, Jong M, Davis S, Wilson D, Fricke T, Resnikoff S. Nearly 1 billion myopes at risk ofmyopia-related sight-threatening conditions by 2050-time to act now. Clin Exp Optom. 2015;98:491-493.

14. Al Wadaani F, Amin T, Ali A, Khan AR. Prevalence and pattern of refractive errors among primary school children in Al Hassa, Saudi Arabia. Glob J Health Sci. 2013;5:125-134.

15. Abushaqara SM, Kazi G, Alrushood A, et al. Prevalence and causes of visual-acuity defect in male school-children in al-khobar area. Saudi Med J. 1991;12:397-402.

16. Aldebasi YH. Prevalence of correctable visual impairment in primary school children in Qassim Province, Saudi Arabia. J Optom. 2014;7:168-176.

17. Al-Rowaily MA. Prevalence of refractive errors among pre-school children at King Abdulaziz Medical City, Riyadh, Saudi Arabia. Saudi J Ophthalmol. 2010;24:45-48.

18. Yamamah GAN, Mohammed AM. Prevalence of visual impairment and refractive errors in children of south Sinai, Egypt. Ophthalmic Epidemiol. 2015;22:357-357.

19. Webber AL, Wood J. Amblyopia: prevalence, natural history, functional effects and treatment. Clin Exp Optom. 2005;88:365-375.

20. World Health Organization, National Institutes of Health. Assessment of the prevalence of visual impairment attributable to refractive error or other causes in school children. In: World Health Organization; 2007.

21. Negrel A, Maul E, Pokharel G, et al. Refractive error study in children: sampling and measurement methods for a multi-country survey. Am J Ophthalmol. 2000;129:421-426. Available from: http://www.who.int/ blindness/causes/RESCProtocol.pdf. Accessed on February 2018.

22. Pokharel GP, Negrel AD, Munoz SR, Ellwein LB. Refractive Error Study in Children: results from Mechi Zone, Nepal. Am J Ophthalmol. 2000;129:436-444.

23. Zhao J, Pan X, Sui R, Munoz SR, Sperduto RD, Ellwein LB. Refractive error study in children: results from Shunyi District, China. Am J Ophthalmol. 2000;129:427-435.

24. Maul E, Barroso S, Munoz SR, et al. Refractive Error Study in Children: results from La Florida, Chile. Am J Ophthalmol. 2000;129:445-454.

25. Dandona R, Dandona L, Srinivas M, Sperduto RD, Ellwein LB. Refractive error in children in a rural population in India. Invest Ophthalmol Vis Sci. 2002;43:615-622. 
26. Murthy GV, Gupta SK, Ellwein LB, et al. Refractive error in children in an urban population in New Delhi. Invest Ophthalmol Vis Sci. 2002; 43:623-631.

27. He MG, Xu JJ, Yin QX, Ellwein LB. Need and challenges of refractive correction in urban Chinese school children. Optom Vis Sci. 2005; 82:229-234

28. Vitale S, Ellwein L, Cotch MF, et al. Prevalence of refractive error in the United States, 1999-2004. Arch Ophthalmol. 2008;126:1111-1119.

29. French AN, Morgan IG, Burlutsky G, Mitchell P, Rose KA. Prevalence and 5-to 6-year incidence and progression of myopia and hyperopia in Australian school children. Ophthalmology. 2013;120:1482-1491.

30. Midelfart A, Kinge B, Midelfart S, Lydersen S. Prevalence of refractive errors in young and middle-aged adults in Norway. Acta Ophthalmol Scand. 2002;80:501-505.

31. Villarreal MG, Ohlsson J, Abrahamsson M, Sjöstrom A, Sjöstrand J. Myopisation: he refractive tendency in teenagers. Prevalence of myopia among young teenagers in Sweden. Acta Ophthalmol Scand. 2000;78:177-181.

32. He MG, Huang WY, Zheng YF, et al. Refractive error and visual impairment in school children in rural southern China. Ophthalmology. 2007;114:374-382.

33. Khader YS, Batayha WQ, Abdul-Aziz SM, Al-Shiekh-Khalil MI. Prevalence and risk indicators of myopia among schoolchildren in Amman, Jordan. East Med Health J. 2006;12:434-439.

34. Wong HB, Machin D, Tan SB, et al. Visual impairment and its impact on health-related quality of life in adolescents. Am J Ophthalmol. 2009;147:505-511.

35. Al-Shaaln FF, Bakrman MA, Ibrahim AM, Wong TY, Saw SM. Prevalence and causes of visual impairment among Saudi adults attending primary health care centers in northern Saudi Arabia. Ann Saudi Med. 2011;31:473-480.

36. Matsumura H, Hirai H. Prevalence of myopia and refractive changes in students from 3 to 17 years of age. Surv Ophthalmol. 1999;44: S109-S115.

37. Giordano L, Friedman D, Repka M, et al. Prevalence of refractive error among preschool children in an urban population: the Baltimore Pediatric Eye Disease Study. Ophthalmology. 2009;116:739-746.

38. Ali A, Talha A, Elmadina AE. Refractive errors status among children examined at optical center in Khartoum state. Sudanese J Ophthalmol. 2016;8:10-13.

39. Mehari ZA, Yimer AW. Prevalence of refractive errors among schoolchildren in rural central Ethiopia. Clin Exp Optom. 2013;96:65-69.

40. Hashemi H, Rezvan F, Beiranvand A, et al. Prevalence of Refractive Errors among High School Students in Western Iran. J Ophthalmic Vis Res. 2014;9:232-239.

41. Norouzirad R, Hashemi H, Yekta A, et al. The prevalence of refractive errors in 6- to 15-year-old schoolchildren in Dezful, Iran. J Curr Ophthalmol. 2015;27:51-55.

42. Caca I, Cingu AK, Sahin A, et al. Amblyopia and refractive errors among school-aged children with low socioeconomic status in southeastern Turkey. J Pediatr Ophthalmol Strabismus. 2013;50:37-43.

43. Ayed T, Sokkah M, Charfi O, et al. [Epidemiologic study of refractive errors in schoolchildren in socioeconomically deprived regions in Tunisia.] J Fr Ophtalmol. 2002;25:712-717. French.

44. Sewunet SA, Aredo KK, Gedefew M. Uncorrected refractive error and associated factors among primary school children in Debre Markos District, Northwest Ethiopia. BMC Ophthalmol. 2014;14:95.

45. Anera RG, Soler M, de la Cruz Cardona J, Salas C, Ortiz C. Prevalence of refractive errors in school-age children in Morocco. Clin Exp Ophthalmol. 2009;37:191-196.

46. Guggenheim JA, Northstone K, McMahon G, et al. Time outdoors and physical activity as predictors of incident myopia in childhood: a prospective cohort study. Invest Ophthalmol Vis Sci. 2012;53:2856-2865.

47. Shah RL, Huang Y, Guggenheim JA, Williams C. Time outdoors at specific ages during early childhood and the risk of incident myopia. Invest Ophthalmol Vis Sci. 2017;58:1158-1166.
48. Pacella R, McLellan J, Grice K, Del Bono EA, Wiggs JL, Gwiazda JE. Role of genetic factors in the etiology of juvenile-onset myopia based on a longitudinal study of refractive error. Optom Vis Sci. 1999;76: $381-386$.

49. Jones LA, Sinnott LT, Mutti DO, Mitchell GL, Moeschberger ML, Zadnik K. Parental history of myopia, sports and outdoor activities, and future myopia. Invest Ophthalmol Vis Sci. 2007;48:3524-3532.

50. Jones-Jordan LA, Sinnott LT, Graham ND, et al. The contributions of near work and outdoor activity to the correlation between siblings in the collaborative longitudinal evaluation of ethnicity and refractive error (CLEERE) study. Invest Ophthalmol Vis Sci. 2014;55:6333-6339.

51. Verhoeven VJM, Hysi PG, Wojciechowski R, et al. Genome-wide metaanalyses of multi-ethnic cohorts identify multiple new susceptibility loci for refractive error and myopia. Nat Genet. 2013;45:314-318.

52. Wojciechowski R, Hysi PG. Focusing in on the complex genetics of myopia. PLoS Genet. 2013;9:e1003442.

53. Kiefer AK, Tung JY, Do CB, et al. Genome-wide analysis points to roles for extracellular matrix remodeling, the visual cycle, and neuronal development in myopia. PLoS Genet. 2013;9:e1003299.

54. Sharanjeet K, Ramli NI, Narayanasamy S. Heredity factor in myopia development among a sample in Klang Valley, Malaysia. Chin Med J. 2012;125:3522-3525.

55. Aldebasi Y. A descriptive study on compliance of spectacle-wear in children of primary schools at Qassim Province, Saudi Arabia. Int J Health Sci. 2013;7:291-299.

56. Congdon N, Zheng MW, Sharma A, et al. Prevalence and determinants of spectacle nonwear among rural Chinese secondary schoolchildren the Xichang pediatric refractive error study report 3. Arch Ophthalmol. 2008;126:1717-1723.

57. Ma XC, Zhou ZQ, Yi HM, et al. Effect of providing free glasses on children's educational outcomes in China: cluster randomized controlled trial. BMJ. 2014;349:g5740.

58. McClure TM, Choi D, Wooten K, Nield C, Becker TM, Mansberger SL. The impact of eyeglasses on vision-related quality of life in American Indian/Alaska natives. Am J Ophthalmol. 2011;151:175-182.

59. Holguin AMC, Congdon N, Patel N, et al. Factors associated with spectacle-wear compliance in school-aged Mexican children. Invest Ophthalmol Vis Sci. 2006;47:925-928.

60. Odedra N, Wedner SH, Shigongo ZS, Nyalali K, Gilbert C. Barriers to spectacle use in Tanzanian secondary school students. Ophthalmic Epidemiol. 2008;15:410-417.

61. Drover JR, Kean PG, Courage ML, et al. Prevalence of amblyopia and other vision disorders in young Newfoundland and Labrador children. Can J Ophthalmol. 2008;43:89-94.

62. Aldebasi YH. Prevalence of amblyopia in primary school children in Qassim province, Kingdom of Saudi Arabia. Middle East Afr J Ophthalmol. 2015;22:86-91.

63. Abolfotouh MA, Badawi I, Faheem Y. Prevalence of amblyopia among schoolboys in Abha city, Asir Region, Saudi Arabia. J Egypt Public Health Assoc. 1994;69:19-30.

64. Williams C, Northstone K, Howard M, Harvey I, Harrad RA, Sparrow JM. Prevalence and risk factors for common vision problems in children: data from the ALSPAC study. Br J Ophthalmol. 2008;92:959-964.

65. Friedman DS, Repka MX, Katz J, et al. Prevalence of amblyopia and strabismus in white and African American children aged 6 through 71 months the Baltimore Pediatric Eye Disease Study. Ophthalmology. 2009;116:2128-2134.

66. He M, Zeng J, Liu Y, Xu J, Pokharel GP, Ellwein LB. Refractive error and visual impairment in urban children in southern China. Invest Ophthalmol Vis Sci. 2004;45:793-799.

67. Goh PP, Abqariyah Y, Pokharel GP, Ellwein LB. Refractive error and visual impairment in school-age children in Gombak district, Malaysia. Ophthalmology. 2005;112:678-685.

68. Robaei D, Huynh SC, Kifley A, Mitchell P. Correctable and non-correctable visual impairment in a population-based sample of 12-year-old Australian children. Am J Ophthalmol. 2006;142:112-118. 


\section{Publish your work in this journal}

Clinical Ophthalmology is an international, peer-reviewed journal covering all subspecialties within ophthalmology. Key topics include: Optometry; Visual science; Pharmacology and drug therapy in eye diseases; Basic Sciences; Primary and Secondary eye care; Patient Safety and Quality of Care Improvements. This journal is indexed on
PubMed Central and CAS, and is the official journal of The Society of Clinical Ophthalmology (SCO). The manuscript management system is completely online and includes a very quick and fair peer-review system, which is all easy to use. Visit http://www.dovepress.com/ testimonials.php to read real quotes from published authors. 